\title{
Convergence and stability of some iterative processes for a class of quasinonexpansive type mappings
}

David Ariza-Ruiz ${ }^{\mathrm{a}, *}$

${ }^{a}$ Department of Mathematical Analysis, University of Seville, Apdo. 1160, 41080-Seville, Spain

This paper is dedicated to Professor Ljubomir Ćirić

Communicated by Professor V. Berinde

\begin{abstract}
Motivated by Dotson's example we consider a certain class of mappings which includes the classes of mappings studied by Zamfirescu, Ćirić, Berinde and others. We prove several new results about convergence of distinct iterative processes in convex metric spaces. Furthermore, we study the stability for this class of mappings in the setting of metric spaces.(C)2012 NGA. All rights reserved.
\end{abstract}

Keywords: Convex metric spaces, Contractive conditions, quasinonexpansive maps, Convergence, Iterative processes, almost $T$-stability.

2010 MSC: Primary 47H09; Secondary 47H10, 54E50, 54H25

\section{Introduction and preliminaries}

Throughout this paper, we denote the set of nonnegative real numbers by $\mathbb{R}^{+}$and by $\operatorname{Fix}(T)$ the set of fixed points of a mapping $T$.

\subsection{Some contractive types}

Let $D$ be a nonempty subset of a metric space $(X, d)$. A mapping $T: D \rightarrow X$ is said to be contractive if there exists a constant $\alpha \in[0,1)$ such that,

$$
d(T x, T y) \leq \alpha d(x, y),
$$

\footnotetext{
* Corresponding author

Email address: dariza-us.es (David Ariza-Ruiz)
} 
for all $x, y \in D$. The well known Banach's fixed point theorem asserts that if $D=X, T$ is contractive and $(X, d)$ is complete, then $T$ has a unique fixed point $p$ in $X$, and for any $x_{0} \in X$ the sequence $\left\{T^{n}\left(x_{0}\right)\right\}$ converges to $p$. This result has been extended by several authors to some classes of mappings by changing the contractive condition (C). For instance, two conditions that can replace (C) in Banach's theorem are the following:

- (Kannan, 25]) There exists $\kappa \in[0,1)$ such that, for all $x, y \in D$,

$$
d(T x, T y) \leq \frac{\kappa}{2}[d(x, T x)+d(y, T y)] .
$$

- (Chatterjea [5]) There exists $\xi \in[0,1)$ such that, for all $x, y \in D$,

$$
d(T x, T y) \leq \frac{\xi}{2}[d(x, T y)+d(y, T x)] .
$$

The conditions $(\mathrm{C}),(\mathrm{K})$ and $(\mathrm{Ch})$ are independent (see [1], 37] and [14]).

In 1972, Zamfirescu [41, combining the conditions (C), (K) and (Ch), obtained a fixed point theorem for the class of mappings $T: X \rightarrow X$ for which there exists $\zeta \in[0,1)$ such that

$$
\begin{aligned}
d(T x, T y) \leq \zeta \max \{d(x, y) & \frac{1}{2}[d(x, T x)+d(y, T y)], \\
& \left.\frac{1}{2}[d(x, T y)+d(y, T x)]\right\} .
\end{aligned}
$$

A mapping satisfying (Z) is commonly called a Zamfirescu mapping. Note that the class of Zamfirescu mappings is a subclass of the class of mappings $T$ satisfying the following condition: there exists $0 \leq h<1$ such that

$$
d(T x, T y) \leq h \max \left\{d(x, y), \frac{1}{2}[d(x, T x)+d(y, T y)], d(x, T y), d(y, T x)\right\} .
$$

This condition was first considered by Ćirić [6] who obtained a fixed point theorem for mappings satisfying $(\mathrm{R})$. Recently, this class of mappings has been studied by Rafiq [34. Notice that every mapping $T$ satisfying $(\mathrm{R})$ is a quasicontraction. The concept of quasicontraction was introduced and investigated by Ćirić [7] in 1971, who obtained an existence fixed point theorem under the following condition: there exists a constan $q \in[0,1)$ such that

$$
d(T x, T y) \leq q \max \{d(x, y), d(x, T x), d(y, T y), d(x, T y), d(y, T x)\} .
$$

Recently, Berinde [2] proved a fixed point result using a new condition, which is independent of (QC). This condition can be stated as follows: there exist two constants $\theta \in[0,1)$ and $L \geq 0$ such that

$$
d(T x, T y) \leq \theta d(x, y)+L d(y, T x)
$$

for all $x, y \in D$. To obtain the uniqueness of the fixed point of a mapping satisfying $(\mathrm{B})$, Berinde considered the following contractive condition, quite similar to $(\mathrm{B})$. There exist two constants $\delta \in[0,1)$ and $L_{1} \geq 0$ such that

$$
d(T x, T y) \leq \delta d(x, y)+L_{1} d(x, T x)
$$

for all $x, y \in D$.

Berinde noticed that the identity mapping on any metric space satisfies $(B)$ but does not $(B])$. With the following example, we prove that both classes of mappings are independent. 
Example 1.1. Let $X=\{a, b\}$ be any set together with the discrete metric $d$. The mapping $T: X \rightarrow X$, given by $T a=b$ and $T b=a$, satisfies $\left(\overrightarrow{B^{\prime}}\right)$ with $\delta \in(0,1)$ arbitrary and $L_{1} \geq 1-\delta$. Indeed,

$$
d(T a, T b)=1 \leq \delta+L_{1}=\delta d(a, b)+L_{1} d(a, b)=\delta d(a, b)+L_{1} d(a, T a)
$$

and, similarly,

$$
d(T b, T a)=1 \leq \delta+L_{1}=\delta d(b, a)+L_{1} d(b, a)=\delta d(b, a)+L_{1} d(b, T b) .
$$

Moreover, $T$ does not satisfy $(\mathrm{B})$, since if there exist $\theta \in[0,1)$ and $L \geq 0$ such that $T$ verifiques $(\mathrm{B})$, then

$$
d(T a, T b) \leq \theta d(a, b)+L d(b, T a),
$$

that is, $1 \leq \theta$, which is a contradiction.

\subsection{Convex metric spaces}

Throughout this paper, we consider a type of metric spaces introduced by Takahashi [39] in 1970. This class of metric spaces, called convex metric spaces, had been used to obtain some generalizations of fixed point results on Banach spaces, for example, by Ćirić for non-self mappings [8, 9, 10, 11, 12, 13.

Definition 1.2. A convex metric space $(X, d, \oplus)$ is a metric space $(X, d)$ together with a convexity mapping $\oplus: X \times X \times[0,1] \rightarrow X$ satisfying

$$
d(z,(1-\lambda) x \oplus \lambda y) \leq(1-\lambda) d(z, x)+\lambda d(z, y)
$$

for all $x, y, z \in X, \lambda \in[0,1]$.

Example 1.3. Obviously, any normed space is a convex metric space. Spaces of hyperbolic type, which were introduced by Goebel and Kirk [18] are convex metric spaces. Hyperbolic spaces in the sense of Reich and Safrir [35] are also convex metric spaces. Other examples of convex metric spaces are Busemann spaces, $C A T(0)$-spaces, Hilbert ball and $\mathbb{R}$-trees (For a deeper discussion we refer the reader to [27]).

Every convex metric space satisfies the following property, which is very important.

Proposition 1.4. If $(X, d, \oplus)$ is a convex metric space, then

$$
d(x,(1-\lambda) x \oplus \lambda y)=\lambda d(x, y) \quad \text { and } \quad d(y,(1-\lambda) x \oplus \lambda y)=(1-\lambda) d(x, y),
$$

for all $x, y \in X$ and $\lambda \in[0,1]$.

As as immediate consequence, we obtain that $1 x \oplus 0 y=x, 0 x \oplus 1 y=y$ and $(1-\lambda) x \oplus \lambda x=\lambda x \oplus(1-\lambda) x=$ $x$.

A nonempty subset $C$ of a convex metric space $(X, d, \oplus)$ is said to be convex if $(1-\lambda) x \oplus \lambda y \in C$ for all $x, y \in C$ and $\lambda \in[0,1]$. A nice feature of our setting is that any convex subset is itself a convex metric space with the restriction of $d$ and $\oplus$ to $C$.

\subsection{Some iterative processes}

Let $D$ be a nonempty subset of a metric space $(X, d)$ and $T: D \rightarrow D$ a self-mapping. Let $x_{0} \in D$ be fixed, we can consider the sequence $\left\{x_{n}\right\}_{n \in \mathbb{N}}$ defined by

$$
x_{n+1}:=T\left(x_{n}\right)=T^{n+1}\left(x_{0}\right), \quad \text { for all } n \in \mathbb{N} .
$$

The sequence defined by $(1.2)$ is known as the Picard iteration.

Let $C$ be a closed subset of a complete metric space $(X, d)$. If $T: C \rightarrow C$ satisfies any of the conditions $(\mathrm{C}),(\mathrm{K}),(\mathrm{Ch}),(\mathrm{Z}),(\mathrm{R}),(\mathrm{QC}),(\mathrm{B})$, then $T$ has at least a fixed point. Moreover, the Picard iteration converges to a fixed point of $T$. However, if any of this conditions is slightly weaker, then the Picard iteration need not converge to a fixed point of the operator $T$. The following trivial example shows this behavior. 
Example 1.5. Let $X=\left\{x_{1}, x_{2}, x_{3}\right\}$, with the discrete metric $d$, and $T: X \rightarrow X$ defined by $T\left(x_{1}\right)=x_{3}$, $T\left(x_{2}\right)=x_{2}$ and $T\left(x_{3}\right)=x_{1}$. It is easy to check that $T$ is nonexpansive, that is, $d\left(T x_{i}, T x_{j}\right) \leq d\left(x_{i}, x_{j}\right)$ for all $i, j \in\{1,2,3\}$. Moreover, the Picard iteration of $T$, with the starting point $x_{1}$ or $x_{3}$, does not converge to $x_{2}$, which is the fixed point of $T$.

In view of above example, we can state that some other iteration processes must be considered. Bearing in mind the iterative processes that exist in the Banach space setting, we shall introduce the most important iterative processes in the convex metric spaces. In order to do this, $C$ will be a convex subset of a convex metric space $(X, d, \oplus)$ and $T: C \rightarrow C$ a mapping.

For any given $x_{0}$ in $C$, the sequence $\left\{x_{n}\right\}_{n \in \mathbb{N}}$ defined by

$$
x_{n+1}=(1-\lambda) x_{n} \oplus \lambda T x_{n}, \quad \text { for all } n \in \mathbb{N},
$$

where $\lambda \in(0,1)$, is called Krasnosel'skij iteration [26].

Mann iteration [28] is essentially an averaged algorithm which generates a sequence recursively

$$
x_{n+1}=\left(1-\alpha_{n}\right) x_{n} \oplus \alpha_{n} T x_{n}, \quad \text { for all } n \in \mathbb{N},
$$

where the initial guess $x_{0} \in C$ and $\left\{\alpha_{n}\right\}_{n \in \mathbb{N}}$ is a sequence in $(0,1)$.

Ishikawa iteration [23] is the following process of two steps: let $x_{0} \in X$ be fixed, consider the sequence $\left\{x_{n}\right\}_{n \in \mathbb{N}}$ defined by

$$
\left\{\begin{array}{l}
y_{n}=\left(1-\beta_{n}\right) x_{n} \oplus \beta_{n} T x_{n}, \\
x_{n+1}=\left(1-\alpha_{n}\right) x_{n} \oplus \alpha_{n} T y_{n}, \quad \text { for all } n \in \mathbb{N},
\end{array}\right.
$$

where $\left\{\alpha_{n}\right\}_{n \in \mathbb{N}}$ and $\left\{\beta_{n}\right\}_{n \in \mathbb{N}}$ are sequence in $[0,1]$.

\section{The class of $\varphi$-quasinonexpansive mappings}

In 1970, Dotson [17] considered the following self-mapping $T: \mathbb{R} \rightarrow \mathbb{R}$, defined by

$$
T x= \begin{cases}\frac{x}{2} \sin \left(\frac{1}{x}\right) & \text { if } x \neq 0, \\ 0 & \text { if } x=0 .\end{cases}
$$

He showed that $T$ satisfies the following property

$$
d(T x, p) \leq \frac{1}{2} d(x, p)
$$

for all $x \in \mathbb{R}, p \in \operatorname{Fix}(T)=\{0\}$. Thus, motivated by this example, we can consider the following class of mappings.

Definition 2.1. Let $D$ be a nonempty subset of a metric space $(X, d)$. We say that $T: D \rightarrow X$ is a $\varphi$-quasinonexpansive mapping if $\operatorname{Fix}(T) \neq \emptyset$ and there exists a function $\varphi: \mathbb{R}^{+} \rightarrow \mathbb{R}^{+}$such that

$$
d(T x, p) \leq \varphi(d(x, p)),
$$

for all $x \in X, p \in \operatorname{Fix}(T)$. 
Recently, Olatinwo have studied intensively contractive conditions that includes as a particular case the class of $\varphi$-nonexpansive mappings (see [29, 31] and many other papers by the same author).

Notice that if we take $\varphi$ as the identity function, we obtain the concept of quasi-nonexpansiveness, which was introduced by Tricomi [40] for real functions and later studied by Diaz and Metcalf [15], [16] and by Dotson [17] for mappings in Banach spaces (see [3, Section 3.5, Section 4.2] for detailed discussion of this and related notions.).

We now proceed to show that the mappings from Subsection 1.1 are in the class of $\varphi$-quasinonexpansive mappings. One can easily show that every contractive mapping is a $\varphi$-quasinonexpansive mapping, with $\varphi(t)=\alpha t$ for $t \in \mathbb{R}^{+}$, using Banach's fixed point theorem. However, Dotson's example shows that the class of $\varphi$-quasinonexpansive mappings properly includes contractive mappings. Moreover, this example can be generalized in such a way that the resulting map is not contractive.

Example 2.2. Let $\varphi: \mathbb{R}+\rightarrow \mathbb{R}^{+}$be a function such that $\varphi(t)<t$ for each $t>0$. Let $X$ be the real line with the usual metric. The mapping $T: \mathbb{R} \rightarrow \mathbb{R}$ defined by

$$
T x= \begin{cases}\varphi(x) \sin \left(\frac{1}{x}\right) & \text { if } x \neq 0, \\ 0 & \text { if } x=0,\end{cases}
$$

is a $\varphi$-quasinonexpansive mapping but $T$ is not a contractive mapping.

Example 2.5 in [3, Page 39] shows that Kannan mappings are also $\varphi$-quasinonexpansive mappings with $\varphi(t):=\frac{\kappa}{2-\kappa} t$, for each $t \in \mathbb{R}^{+}$. Using a similar argument, one can prove that every Chatterjea mapping is a $\varphi$-quasinonexpansive mapping, with $\varphi(t)=\xi t$ for $t \in \mathbb{R}^{+}$. Thus, we can deduce that every Zamfirescu mapping is a $\varphi$-quasinonexpansive mapping with $\varphi(t):=\zeta t$, for each $t \in \mathbb{R}^{+}$. The following result, which is implicitly included in [3], shows that this fact is still true for a more general class of mappings.

Proposition 2.3. [3] Let $(X, d)$ be a complete metric space. If $T: X \rightarrow X$ satisfies (QC), then $T$ is a $\varphi$-quasinonexpansive mapping, with $\varphi(t):=\max \left\{q, \frac{q}{1-q}\right\} t$.

Proof. Ćirić [7] proved that $T$ has a unique fixed point $p$ in $X$. Taking $y=p$ in (QC) we get

$$
\begin{aligned}
d(T x, p) & =d(T x, T p) \\
& \leq q \max \{d(x, p), d(x, T x), d(p, T p), d(x, T p), d(p, T x)\} \\
& \leq q \max \{d(x, p), d(x, p)+d(p, T x), d(p, T x)\},
\end{aligned}
$$

for each $x$ in $X$. Since $0 \leq q<1$, we deduce

$$
d(T x, p) \leq \max \left\{q, \frac{q}{1-q}\right\} d(x, p),
$$

for every $x \in X$.

In the case of mappings satisfying $(\bar{R})$, we can obtain a better function $\varphi$.

Proposition 2.4. [3] Let $(X, d)$ be a complete metric space. If $T: X \rightarrow X$ satisfies $(\mathbb{R}$, then $T$ is a $\varphi$-quasinonexpansive mapping, with $\varphi(t):=h t$.

Proof. By Ćirić [7], we know that $T$ has a unique fixed point in $X$, say $p$. If we take $y=p$ in $(\mathrm{R})$ we get

$$
\begin{aligned}
d(T x, p) & =d(T x, T p) \\
& \leq h \max \left\{d(x, p), \frac{1}{2}[d(x, T x)+d(p, T p)], d(x, T p), d(p, T x)\right\} \\
& \leq h \max \left\{d(x, p), \frac{1}{2}[d(x, p)+d(p, T x)]\right\}
\end{aligned}
$$


for each $x$ in $X$. Hence,

$$
d(T x, p) \leq \max \left\{h, \frac{h}{2-h}\right\} d(x, p)=h d(x, p),
$$

for every $x \in X$, because $0 \leq h<1$.

A trivial verification shows that if $T$ has at least one fixed point and satisfies $(\vec{B})$, then $T$ is a $\varphi$ nonexpansive mapping, with $\varphi(t)=\delta t$ for each $t \in \mathbb{R}^{+}$.

We must notice that there exist other classes of mappings which belong to the class of $\varphi$-quasinonexpansive mappings. For example, Jaggi [24] proved the following fixed point theorem.

Theorem 2.5. Let $T$ be a continuous selfmap defined on a complete metric space $(X, d)$. Suppose that $T$ satisfies the following contractive condition:

$$
d(T x, T y) \leq \alpha \frac{d(x, T x) d(y, T y)}{d(x, y)}+\beta d(x, y)
$$

for all $x, y \in X, x \neq y$, and for some $\alpha, \beta \in[0,1)$ with $\alpha+\beta<1$, then $T$ has a unique fixed point in $X$.

A trivial verification shows that if $T$ satisfies the assumptions of Jaggi's theorem, then $T$ is a continuous $\varphi$-quasinonexpansive mapping, with $\varphi(t):=\beta t$ for each $t \in \mathbb{R}^{+}$.

\section{Convergence results}

Here and subsequently, $\Phi$ denotes the family of functions $\varphi: \mathbb{R}^{+} \rightarrow \mathbb{R}^{+}$such that $\varphi$ is continuous and $\varphi(t)<t$ for all $t>0$. Before we discuss our results, we state an elementary numerical result.

Lemma 3.1. Let $\left\{\lambda_{n}\right\}_{n \in \mathbb{N}}$ be a real sequence in $[0,1]$ and let $\left\{d_{n}\right\}_{n \in \mathbb{N}}$ be a sequence of nonnegative real numbers such that

$$
d_{n+1} \leq\left(1-\lambda_{n}\right) d_{n}+\lambda_{n} \varphi\left(d_{n}\right) \quad \text { for all } n \in \mathbb{N},
$$

where $\varphi \in \Phi$. If $\left\{\lambda_{n}\right\}_{n \in \mathbb{N}}$ converges to $\lambda \in(0,1]$, then we have $\lim _{n \rightarrow \infty} d_{n}=0$.

Proof. Since $\varphi(t) \leq t$ for all $t \in \mathbb{R}^{+}$, we get that $\left\{d_{n}\right\}_{n \in \mathbb{N}}$ is nonincreasing and, therefore, convergent to a nonnegative real number $d$. We shall show that $d=0$. In order to do this, we assume that $d>0$ and we obtain a contradiction as follows. Since $\lambda_{n} \rightarrow \lambda \in(0,1]$, as $n \rightarrow \infty$, taking limits in (3.1) we have that

$$
d \leq(1-\lambda) d+\lambda \varphi(d)<(1-\lambda) d+\lambda d=d,
$$

which is a contradiction. Therefore, $d=0$, that is, $\lim _{n \rightarrow \infty} d_{n}=0$.

Remark 3.2. The above result still holds if it is just assumed that $\varphi$ is a function satisfying $\varphi(t)<t$ for each $t>0$ and

$$
\text { if }\left\{t_{n}\right\}_{n \in \mathbb{N}} \searrow t \text {, then } \liminf _{n \rightarrow \infty} \varphi\left(t_{n}\right) \leq \varphi(t) .
$$

We now prove the convergence of the Mann iteration process on a convex metric space, when the operator $T$ is assumed to be only $\varphi$-quasinonexpansive.

Theorem 3.3. Let $C$ be a convex subset $C$ of a convex metric space $(X, d, \oplus)$. Assume that $T: C \rightarrow C$ is a $\varphi$-quasinonexpansive mapping with $\varphi \in \Phi$. Let $\left\{\alpha_{n}\right\}_{n \in \mathbb{N}}$ be a real sequence in $[0,1]$ such that $\left\{\alpha_{n}\right\}_{n \in \mathbb{N}}$ converges to some positive real number. Then, for any $x_{0}$ in $X$, the sequence $\left\{x_{n}\right\}_{n \in \mathbb{N}}$ defined by (1.4) converges to the unique fixed point of $T$. 
Proof. Let us first prove that $T$ has a unique fixed point in $C$. Suppose that $p, q \in \operatorname{Fix}(T)$, with $p \neq q$. Using (2.1) and the property of $\varphi$, we obtain

$$
d(q, p)=d(T q, p) \leq \varphi(d(q, p))<d(q, p),
$$

which is a contradiction. Let $x_{0} \in C$ be arbitrary. Now, we shall prove that the Mann iteration $\left\{x_{n}\right\}_{n \in \mathbb{N}}$ converges to $p$, where $\operatorname{Fix}(T)=\{p\}$. Since

$$
\begin{aligned}
d\left(x_{n+1}, p\right) & =d\left(\left(1-\alpha_{n}\right) x_{n} \oplus \alpha_{n} T x_{n}, p\right) \\
& \leq\left(1-\alpha_{n}\right) d\left(x_{n}, p\right)+\alpha_{n} d\left(T x_{n}, p\right) \\
& \leq\left(1-\alpha_{n}\right) d\left(x_{n}, p\right)+\alpha_{n} \varphi\left(d\left(x_{n}, p\right)\right),
\end{aligned}
$$

for every $n \in \mathbb{N}$, by Lemma 3.1 we deduce that $\left\{x_{n}\right\}_{n \in \mathbb{N}}$ converges to $p$.

Remark 3.4. Clearly, if we take $\left\{\alpha_{n}\right\}_{n \in \mathbb{N}}$ as a constant sequence in $(0,1]$, we get a result about the convergence of Krasnosel'skij iteration.

Notice that if in the above result we take $\alpha_{n}=1$ for all $n \in \mathbb{N}$, we obtain a result about the convergence of the Picard iteration process. Moreover, this result still holds if it is just assumed that $C$ is a nonempty subset of a metric space $(X, d)$.

Corollary 3.5. Let $C$ be a nonempty subset of a metric space $(X, d)$. If $T: C \rightarrow C$ is a $\varphi$-quasinonexpansive mapping, with $\varphi: \mathbb{R}^{+} \rightarrow \mathbb{R}^{+}$being a continuous function such that $\varphi(t)<t$ for all $t>0$, then the sequence $\left\{x_{n}\right\}_{n \in \mathbb{N}}$ defined by $(1.2)$ converges to the unique fixed point of $T$, for any $x_{0}$ in $X$.

Using a similar argument as in Theorem 3.3, we can establish a strong convergence of the Mann iteration process to a common fixed point for a finite family of $\varphi$-quasinonexpansive mappings in convex metric spaces.

Theorem 3.6. Let $C$ be a convex subset $C$ of a convex metric space $(X, d, \oplus)$. Let $T_{i}: C \rightarrow C$ be a finite family of $\varphi_{i}$-quasinonexpansive mappings, $i=1, \ldots, N$, with $\varphi_{i} \in \Phi$ and $\cap_{i=1}^{N} F i x\left(T_{i}\right) \neq \emptyset$. Let $\left\{\alpha_{n}\right\}_{n \in \mathbb{N}}$ be a real sequence in $[0,1]$ such that $\left\{\alpha_{n}\right\}_{n \in \mathbb{N}}$ converges to some positive real number. Then, for any $x_{0}$ in $X$, the sequence $\left\{x_{n}\right\}_{n \in \mathbb{N}}$ defined by

$$
x_{n+1}=\left(1-\alpha_{n}\right) x_{n} \oplus \alpha_{n} T_{n(\bmod N)} x_{n}, \quad \text { for all } n \in \mathbb{N},
$$

converges to a common fixed point of $\left\{T_{i}\right\}_{i=1}^{N}$.

Proof. Note that if $p \in \cap_{i=1}^{N} \operatorname{Fix}\left(T_{i}\right)$, then we have

$$
\begin{aligned}
d\left(x_{n+1}, p\right) & \leq d\left(\left(1-\alpha_{n}\right) x_{n} \oplus \alpha_{n} T_{n(\bmod N)} x_{n}, p\right) \\
& \leq\left(1-\alpha_{n}\right) d\left(x_{n}, p\right) \oplus \alpha_{n} d\left(T_{n(\bmod N)} x_{n}, p\right) \\
& \leq\left(1-\alpha_{n}\right) d\left(x_{n}, p\right) \oplus \alpha_{n} \varphi_{n(\bmod N)}\left(d\left(x_{n}, p\right)\right) \\
& \leq\left(1-\alpha_{n}\right) d\left(x_{n}, p\right) \oplus \alpha_{n} \varphi\left(d\left(x_{n}, p\right)\right),
\end{aligned}
$$

for all $n \in \mathbb{N}$, where $\varphi:=\max \left\{\varphi_{1}, \ldots, \varphi_{N}\right\}$. Moreover, $\varphi \in \Phi$ because each $\varphi_{i} \in \Phi$.

We can extend Theorem 3.3 to the Ishikawa iteration process. We omit the proof because it is similar to the proof of Theorem 3.3 .

Theorem 3.7. Let $C$ be a convex subset $C$ of a convex metric space $(X, d, \oplus)$. Assume that $T: C \rightarrow C$ is a $\varphi$-quasinonexpansive mapping, with $\varphi \in \Phi$. Let $\left\{\alpha_{n}\right\}_{n \in \mathbb{N}}$ and $\left\{\beta_{n}\right\}_{n \in \mathbb{N}}$ be two real sequences in $[0,1]$ such that $\left\{\alpha_{n} \beta_{n}\right\}_{n \in \mathbb{N}}$ converges to some positive real number. Then, for any $x_{0}$ in $X$, the sequence $\left\{x_{n}\right\}_{n \in \mathbb{N}}$ defined by (1.5) converges to the unique fixed point of $T$. 
Remark 3.8. It is easy to check that our results remain true if $\varphi: \mathbb{R}^{+} \rightarrow \mathbb{R}^{+}$satisfies $(\mathrm{P})$ and $\varphi(t)<t$ for all $t>0$.

Olatinwo [31] proved the convergence of Ishikawa iteration for mappings $T$ in the setting of Banach spaces satisfying the following condition:

$$
\|T x-T y\| \leq \frac{\psi(\|T x-x\|)+a\|x-y\|}{1+M\|x-T x\|} \quad \text { for all } x, y \in X,
$$

where $a \in[0,1), M \geq 0$ and $\psi: \mathbb{R}^{+} \rightarrow \mathbb{R}^{+}$is a monotone increasing function such that $\psi(0)=0$. Notice that the above condition implies the $\varphi$-nonexpansiveness with $\varphi(t)=a t$.

\section{On stability for $\varphi$-quasinonexpansive mappings}

For the convenience of the reader, we begin this section with two definitions about stability of a general iteration process.

Definition 4.1. Let $(X, d)$ be a metric space, $T: X \rightarrow X$ a self-mapping of $X$. Let $\left\{x_{n}\right\}_{n \in \mathbb{N}} \subset X$ be the sequence generated by an iteration procedure involving $T$ which is defined by

$$
x_{n+1}=f\left(T, x_{n}\right) \quad \text { for } n \in \mathbb{N},
$$

where $x_{0} \in X$ is the initial approximation and $f$ is some function. Suppose $\left\{x_{n}\right\}_{n \in \mathbb{N}}$ converges to a fixed point $p$ of $T$. Let $\left\{y_{n}\right\}_{n \in \mathbb{N}} \subset X$ and set

$$
\varepsilon_{n}:=d\left(y_{n+1}, f\left(T, y_{n}\right)\right) \quad \text { for } n \in \mathbb{N} .
$$

Then,

$\left(D_{1}\right)$ the iteration process (4.1) is said to be $T$-stable or stable with respect to $T$ if $\lim _{n \rightarrow \infty} \varepsilon_{n}=0$ implies $\lim _{n \rightarrow \infty} y_{n}=p$.

$\left(D_{2}\right)$ the iteration process 4.1$]$ is said to be almost $T$-stable or almost stable with respect to $T$ if $\sum_{n \in \mathbb{N}} \varepsilon_{n}<\infty$ implies $\lim _{n \rightarrow \infty} y_{n}=p$.

The concept of stability of a fixed point iteration procedure seems to be due to Ostrowski, as mentioned by Rhoades [38, but has been systematically studied by Harder [19] in her Ph.D. thesis and published in the papers of Harder and Hicks [20], 21] (see [3] for more details). Recently, Rezapour et al. [36] have studied almost $T$-stability of Mann iteration for $\varphi$-quasinonexpansive mappings with the following additional assumptions for $\varphi$ :

(i) $\varphi$ is increasing;

(ii) $\varphi(0)=0$;

(iii) $\varphi(t)<t$ for all $t>0$;

(iv) $\varphi$ is convex;

(v) $\varphi(s+t) \leq \varphi(s)+\varphi(t)$ for all $s, t \in \mathbb{R}^{+}$.

Remark 4.2. It is easy to check that an iterative process (4.1) which is $T$-stable is almost $T$-stable. Osilike [33] gave an example showing that an iterative process which is almost $T$-stable may fail to be $T$-stable

In the paper [30] by Olatinwo, the reader can find an excellent introduction and some interesting comments about several stability results established in metric spaces and normed linear spaces.

During the proof of the main result of this section, we shall need the following result. 
Lemma 4.3. Suppose that $\left\{a_{n}\right\}_{n \in \mathbb{N}}$ and $\left\{b_{n}\right\}_{n \in \mathbb{N}}$ are two sequences of nonnegative numbers such that

$$
a_{n+1} \leq \varphi\left(a_{n}\right)+b_{n}
$$

for all $n \in \mathbb{N}$, where $\varphi \in \Phi$. If $\sum_{n \in \mathbb{N}} b_{n}$ converges, then $\lim _{n \rightarrow \infty} a_{n}=0$.

Proof. Using (4.2), we get

$$
a_{n+m+1} \leq a_{n+m}+b_{n+m} \leq \cdots \leq a_{n}+\sum_{i=n}^{n+m} b_{i},
$$

for all $n, m \in \mathbb{N}$, since $\varphi(t) \leq t$ for all $t \geq 0$. Then,

$$
\limsup _{m \rightarrow \infty} a_{m} \leq a_{n}+\sum_{i=n}^{\infty} b_{i},
$$

for every $n \in \mathbb{N}$, which implies that

$$
\limsup _{m \rightarrow \infty} a_{m} \leq \liminf _{n \rightarrow \infty} a_{n} .
$$

Therefore, there exists $a \in \mathbb{R}^{+}$such that $a_{n} \rightarrow a$, as $n \rightarrow \infty$. Assume that $a>0$. Since $\sum_{n \in \mathbb{N}} b_{n}$ converges, $\left\{b_{n}\right\}_{n \in \mathbb{N}}$ converges to 0 . Thus, taking limits in 4.2), we get $a \leq \varphi(a)<a$, which is a contradiction. Therefore, $a=0$.

Remark 4.4. A minor change in the proof actually shows that Lemma 4.3 is still true if $\varphi$ satisfies the following property instead of continuity.

$$
\text { If } \lim _{n \rightarrow \infty} t_{n}=t \text {, then } \liminf _{n \rightarrow \infty} \varphi\left(t_{n}\right) \leq \varphi(t) .
$$

Notice that $\left(\mathrm{P}^{\prime}\right)$ implies $(\mathrm{P})$.

We now discuss the question of almost stability of Picard's iteration process.

Theorem 4.5. Let $(X, d)$ be a complete metric space and $T: X \rightarrow X$ be a $\varphi$-quasinonexpansive mapping, with $\varphi \in \Phi$. Let $p$ be the unique fixed point of $T$. Let $x_{0} \in X$ and $x_{n+1}=T x_{n}, n \in \mathbb{N}$, be the Picard iteration. Let $\left\{y_{n}\right\}_{n \in \mathbb{N}} \subset X$ and define $\left\{\varepsilon_{n}\right\}_{n \in \mathbb{N}}$ by

$$
\varepsilon_{n}:=d\left(y_{n+1}, T y_{n}\right), \quad \text { ffor } n \in \mathbb{N} .
$$

If $\sum_{n \in \mathbb{N}} \varepsilon_{n}<\infty$, then $\lim _{n \rightarrow \infty} y_{n}=p$. That is, the Picard iteration is almost stable with respect to $T$.

Proof. Notice that Corollary 3.5 states that the Picard iteration converges to $p$, the unique fixed point of $T$. Since

$$
d\left(y_{n+1}, p\right) \leq d\left(y_{n+1}, T y_{n}\right)+d\left(T y_{n}, p\right) \leq \varepsilon_{n}+\varphi\left(d\left(y_{n}, p\right)\right)
$$

for each $n \in \mathbb{N}$ and $\varphi(t) \leq t$ for $t \geq 0$, then $d\left(y_{n+1}, p\right) \leq \varepsilon_{n}+d\left(y_{n}, p\right)$ for all $n \in \mathbb{N}$. By Lemma 4.3 , with $a_{n}=d\left(y_{n}, p\right)$ and $b_{n}=\varepsilon_{n}$, we deduce that

$$
\lim _{n \rightarrow \infty} d\left(y_{n}, p\right)=0
$$

that is, $\left\{y_{n}\right\}$ converges to $p$.

Remark 4.6. We shall not study the analogues of Theorem 4.5 for Mann, Ishikawa, or any other iteration process because, if one obtains stability for a map using Picards iteration, there is no point in considering any other more complicated iteration procedure. 
If $\varphi(t)=\varrho t$ for all $t \in \mathbb{R}^{+}$, with $0<\varrho<1$, then we can obtain a result of stability. This was proved by Bosede and Rhoades [4].

Remark 4.7. Osilike [32] established stability of Picard, Kirk, Mann and Ishikawa iterations for mappings $T$ having a fixed point and satisfying $\left(\mathrm{B}^{\prime}\right]$. Imoru and Olatinwo [22] generalized this condition by replacing $\left(\mathrm{B}^{\prime}\right)$ with

$$
d(T x, T y) \leq \delta d(x, y)+\psi(d(x, T x))
$$

where $0 \leq \delta<1$ and $\psi: \mathbb{R}^{+} \rightarrow \mathbb{R}^{+}$is monotone increasing with $\psi(0)=0$. Notice that the above conditions imply the $\varphi$-quasinonexpansiveness (see [4]).

Acknowledgements: The author wishes to express his sincere thanks to the referee for his suggestions regarding the improvement of the paper. The author is grateful to Professor Genaro López-Acedo for his careful reading and valuable suggestions. This work is partly supported by Junta de Andalucía, Grant FQM-3543.

\section{References}

[1] D. Ariza-Ruiz, A. Jiménez-Melado, Genaro López-Acedo, A fixed point theorem for weakly Zamfirescu mappings, Nonlinear Analysis 74 (2011), 1628-1640. 1.1

[2] V. Berinde, Approximation fixed points of weak contractions using the Picard iteration, Nonlinear Analysis Forum 9 (2004), no. 1, 43-53. 1.1

[3] V. BERINDE, Iterative approximation of fixed points, Springer-Verlag 2007. 2, $2,2.3,2.4,4$

[4] A.O. Bosede, B.E. Rhondes, Stability of Picard and Mann iteration for a general class of functions, J. Adv. Math. Studies 3 (2010), no. 2, 23-25. 4, 4.7

[5] S.K. Chatterjea, Fixed-point theorems, C. R. Acad. Bulgare Sci. 25 (1972), 727-730. 1.1

[6] LJ.B. ĆIRIĆ, Generalized contractions and fixed-point theorems, Publ. Inst. Math. (Beograd) (N.S.) bf 12(26) (1971), 19-26. 1.1

[7] Lj.B. Ćirić, A generalization of Banach's contraction principle, Proc. Am. Math. Soc. 45 (1974), 267-273. 1.1 2 2, 2

[8] LJ.B. Ćirić, A remark on Rhoades fixed point theorem for non-self mappings, Int. J. Math. Math. Sci. 16 (1993), $397-400$. 1.2

[9] LJ.B. Ćirić, Quasi-contraction non-self mappings on Banach spaces, Bull. Acad. Serbe Sci. Arts 23 (1998), 25-31. 1.2

[10] LJ.B. ĆIRIć, Contractive-type non-self mappings on metric spaces of hyperbolic type, J. Math. Anal. Appl. 317 (2006), 28-42. 1.2

[11] LJ.B. ĆIRIĆ, Non-self mappings satisfying non-linear contractive condition with applications, Nonlinear Analysis 71 (2009), $2927-29351.2$

[12] LJ.B. ĆIRIĆ, N. CAKIĆ, On Common fixed point theorems for non-self hybrid mappings in convex metric spaces, Appl. Math. Comput. 208 (2009) 90-97. 1.2

[13] LJ.B. Ćirić, J.S. Ume, Multi-valued non-self mappings on convex metric spaces, Nonlinear Anal. 60 (2005), $1053-1063$. 1.2

[14] P. Collaço, J.C.E Silva, A complete comparison of 25 contraction conditions, Nonlinear Anal. TMA 30 (1997), no. 1, 471-476. 1.1

[15] J.B. Diaz, F.T. Metcalf, On the structure of the set of subsequential limit points of successive approximations, Bull. Amer. Math. Soc. 73 (1967), 516-519. 2

[16] J.B. Diaz, F.T. Metcalf, On the set of subsequential limit points of successive approximations, Trans. Amer. Math. Soc. 135 (1969), 459-485. 2

[17] W.G.Jr. Dotson, On the Mann iterative process, Trans. Amer. Math. Soc. 149 (1970), 65-73. 2, 2,

[18] K. Goebel, W.A. KIRK, Iteration processes for nonexpansive mappings, in Topological Methods in Nonlinear Functional Analysis (S.P. Singh and S. Thomier, eds.), Contemporary Mathematics 21, Amer. Math. Soc. Providence, (1983), 115-123. 1.3

[19] A.M. HARDER, Fixed point theory and stability resuts for fixed points iteration procedures, PhD Thesis University of Missouri-Rolla (1987). 4

[20] A.M. Harder, T.L. Hicks, A stable iteration procedure for nonexpansive mappings, Math. Japon. 33 (1988), no.5, 687-692. 4

[21] A.M. HARDer, T.L. Hicks, Stability results for fixed point iteration procedures, Math. Japon. 33 (1988), no.5, 693-706. 4

[22] C.O. Imoru, M.O. Olatinwo, On the stability of Picard and Mann iteration processes, Carpathian J. Math. 19 (2003), 155-160. 4.7 
[23] S. IshIKAWA, Fixed point and iteration of a nonexpansive mapping in a Banach space, Proc. Amer. Math. Soc. 44 (1976), 147-150. 1.3

[24] D.S. JAGGI, Some unique fixed point theorems, Indian Journal of Pure and Applied Mathematics 8 (1977), no. 2, 223-230. 2

[25] R. Kannan, Some results on fixed points, Bull. Calcutta Math. Soc. 60 (1968), 71-76. 1.1

[26] Krasnosel'skiJ, Two remarks on the method of successive approximations (Russian), Uspehi Mat. Nauk 10 (1955), no. 1(63), 123-127. 1.3

[27] L. Leusţean, Proof mining in fixed point theory and ergodic theory, Oberwolfach Prepints OWP 2009-05, Mathematisches Forschungsinstitut Oberwolfach, Germany (2009), 71pp. 1.3

[28] W.R. Mann, Mean value methods in iteration, Proceedings of American Mathematical Society 4 (1953), no. 3, 506-510. 1.3

[29] M.O. Olatinwo, Some stability results for nonexpansive and quasi-nonexpansive operators in uniformly convex Banach spaces usign the Ishikawa iteration process, Carpathian J. Math. 24 (2008), no. 1, 82-87. 2

[30] M.O. Olatinwo, Some stability results for two hybrid fixed point iterative algorithms of Kir-Ishikawa and Kirk-Mann type, J. Adv. Math. Studies 1 (2008), no. 1-2, 87-96. 4

[31] M.O. Olatinwo, Convergence and stability results for some iterative schemes, Acta Universitatis Apulensis 26 (2011), 225-236. 23

[32] M.O. OsiLIKE, Stability results for fixed point iteration procedures, J. Nigerian Math. Soc. 14/15 (1995/96), 17-28. 4.7

[33] M.O. OsILIKE, Stability of the Mann and Ishikawa iteration procedures for $\phi$-strong pseudocontractions an nonlinear equation of the $\phi$-strongly accretive type, J. Math. Anal. Appl. 227 (1998) 319-334. 4.2

[34] A. RAFIQ, Fixed points of Ćirić quasi-contractive operators in normed spaces, Mathematical Communications 11 (2006), 115-120. 1.1

[35] S. Reich, I. SAFriR, Nonexpansive iterations in hyperbolic spaces, Nonlinear Anal. 15 (1990), 537-558. 1.3

[36] Sh. Rezapour, R.H. Haghi, B.E. Rhoades, Some results about T-stability and almost T-stability, Fixed Point Theory 12 (2011), no.1, 179-186. 4

[37] B.E. RhoAdes, A comparison of various definitions of contractive mappings, Trans. Amer. Math. Soc. 226 (1977), 257-290. [1.1

[38] B.E. RhOADEs, A biased discussion of fixed point theory, Carpathian J. Math. 23 (2007), no.1-2, 11-26. 4

[39] W. TAkAhashi, A convexity in metric space and nonexpansive mapping I, Kodai Math. Sem. Rep. 22 (1970), 142-149. 1.2

[40] F. TRICOMI, Una teorema sulla convergenza delle successioni formate delle successive iterate di una funzione di una variabile reale, Giorn. Mat. Bataglini 54 (1916), 1-9. 2

[41] T. ZAmfirescu, Fixed point theorems in metric spaces, Arch. Math. 23 (1972), 292-298. 1.1 